\title{
Investigating the Dose-Related Effects of Video Game Trunk Control Training in Chronic Stroke Patients With Poor Sitting Balance
}

\author{
Hyun Young Kim, MD, Hyun Im Moon, MD, You Hyeon Chae, MD, Tae Im Yi, MD \\ Department of Rehabilitation Medicine, Bundang Jesaeng General Hospital, Seongnam, Korea
}

\begin{abstract}
Objective To investigate the dose-related effect of trunk control training (TCT) using Trunk Stability Rehabilitation Robot Balance Trainer (TSRRBT) in chronic stroke patients with poor sitting balance.

Methods This was a retrospective study of 38 chronic stroke patients with poor sitting balance that underwent TCT with TSRRBT. The participants were assigned either to the low-dose training (LDT) group $(\mathrm{n}=18)$ or to the highdose training (HDT) group $(\mathrm{n}=20)$. In addition to the conventional rehabilitation therapy, the LDT group received 5 sessions of TSRRBT intervention per week, whereas the HDT group received 10 sessions of TSRRBT intervention per week. The outcome measures were the scores on the Trunk Impairment Scale (TIS) and its subscales, Berg Balance Scale (BBS), Functional Ambulation Classification (FAC), and the Korean version of Modified Barthel Index (K-MBI). All outcome measures were assessed before the training and at the end of the 4-week training. Results After the 4-week intervention, TIS, BBS, FAC, and K-MBI scores showed improvement in both LDT and HDT groups. Furthermore, the improvements in TIS scores and its subscales were significantly greater in the HDT group than in the LDT group $(\mathrm{p}<0.05)$.

Conclusion TCT using TSRRBT could be an additional treatment for the conventional rehabilitation therapy of chronic stroke patients with poor sitting balance. HDT may provide more beneficial effects on improving patients' sitting balance than LDT.
\end{abstract}

Keywords Chronic stroke, Trunk control, Sitting balance, Biofeedback

\section{INTRODUCTION}

Stroke is a pervasive worldwide health care problem that is common, serious, and disabling [1]. Patients with stroke commonly have trunk malalignment, difficulty in maintaining balance, and asymmetry of weight distribution $[2,3]$. Stroke patients show a significantly reduced ability to control their trunk compared with healthy indi-

Received July 26, 2017; Accepted October 18, 2017

Corresponding author: Tae Im Yi

Department of Rehabilitation Medicine, Bundang Jesaeng General Hospital, 20 Seohyeon-ro 180beon-gil, Bundang-gu, Seongnam 13590, Korea. Tel: +82-31-779-0063, Fax: +82-31-779-0635, E-mail: taeim@hanmail.net

ORCID: Hyun Young Kim (http://orcid.org/0000-0003-4920-8374); Hyun Im Moon (http://orcid.org/0000-0003-3430-1824); You Hyeon Chae (http:// orcid.org/0000-0002-3403-0975); Tae Im Yi (http://orcid.org/0000-0001-6369-4677).

(c) This is an open-access article distributed under the terms of the Creative Commons Attribution Non-Commercial License (http://creativecommons.org/ licenses/by-nc/4.0) which permits unrestricted noncommercial use, distribution, and reproduction in any medium, provided the original work is properly cited. Copyright (c) 2018 by Korean Academy of Rehabilitation Medicine 
viduals. Balance impairment in the sitting position is also a common clinical problem after a stroke. Sitting balance involves the ability to reach for objects located both within and beyond arm's length in personal daily tasks, such as showering, toileting, and dressing [4]. In activities of daily living, trunk performance for functional tasks is closely related to maintaining balance in the sitting position [5].

Intensive and repetitive practice of specific functional tasks is important for recovery after a stroke. This situation has made stroke rehabilitation a labor-intensive process; as a result, robot-assisted therapy, which has the advantage of delivering high-dose and high-intensity training, has grown rapidly [6]. Although intensive practice is very important to induce a therapeutic effect, patients tend to lose interest in repetitive treatments, thereby reducing treatment effectiveness [7]. Computer game-based biofeedback treatments were developed and applied in the rehabilitation of stroke patients to address this problem [8-10]. Positive results from studies involving computer game-based biofeedback systems, particularly BalPro [11] and Tetrax [12], are widely reported. Recently, many studies were conducted on robotic rehabilitation systems with video games. Most of them are focused on upper limb function, with some examining gait function. However, robotic rehabilitation combined with video games for trunk control training has rarely been investigated.

The purpose of this study is to investigate whether trunk control training (TCT) in the sitting position with a video game-based biofeedback system would improve sitting balance and the functional outcomes of chronic stroke patients. Another purpose of the study was to determine whether the effects of the training described above were influenced by the amount of treatments or treatment dose.

\section{MATERIALS AND METHODS}

\section{Participants}

This retrospective study included chronic stroke patients who were hospitalized at the Department of Rehabilitation Medicine in Bundang Jesaeng General Hospital between December 2014 and May 2017. The inclusion criteria were as follows: (1) first stroke event confirmed using MRI or CT; (2) history of stroke onset of 6 months or more; (3) poor sitting balance; and (4) completion of four weeks of TCT with the Trunk Stability Rehabilitation Robot Balance Trainer (TSRRBT). The exclusion criteria were as follows: (1) age <18 years; (2) inability to continuously perform TCT for 30 minutes; (3) communication problems; (4) presence of a neurological disease other than stroke, such as Parkinson disease, hypoxic brain injury, or diffuse axonal injury; (5) visual or vestibular system disease affecting balance; or (6) a musculoskeletal disorder, such as fracture or low back pain.

This study was approved by the Institutional Review Board of Bundang Jesaeng General Hospital (No. RM1708).

\section{Interventions}

During the 4-week intervention period, all patients received a conventional stroke rehabilitation therapy with an additional TCT program. The conventional treatment consisted of physical and occupational therapies, including speech and language, as well as cognitive therapies, as needed. TCT was conducted once or twice daily. Through a chart review, patients who underwent 5 sessions of TCT per week were classified as the low-dose training (LDT) group, whereas those who attended 10 sessions per week were classified as the high-dose training (HDT) group.

The TCT program was conducted using TSRRBT (Man\&tel, Gumi, Korea). The TSRRBT consists of a foothold, robot arm, robot chair, monitor sections, and a mechanical body frame (Fig. 1). Pressure sensors were present in the foothold and chair sections. The sensors processed the patient's weight distribution, and the software translated it into a movement of the cursor on the screen. The weight shifting of the patient's torso in the anterior-posterior plane represented the vertical movement of the cursor on the monitor, whereas the weight shifting in the left-right plane represented the horizontal movement of the cursor. Patients played the games by moving the cursor on the screen. If the cursor was placed at a specific location on the monitor for several seconds (different for each game), the patient earned points. Four types of games (balloon popping, fruit catching, archery, and basketball) were included in the TSRRBT intervention (Fig. 2).

The patients and the supervisor learned the TSRRBT protocol from the therapist before the intervention, and 


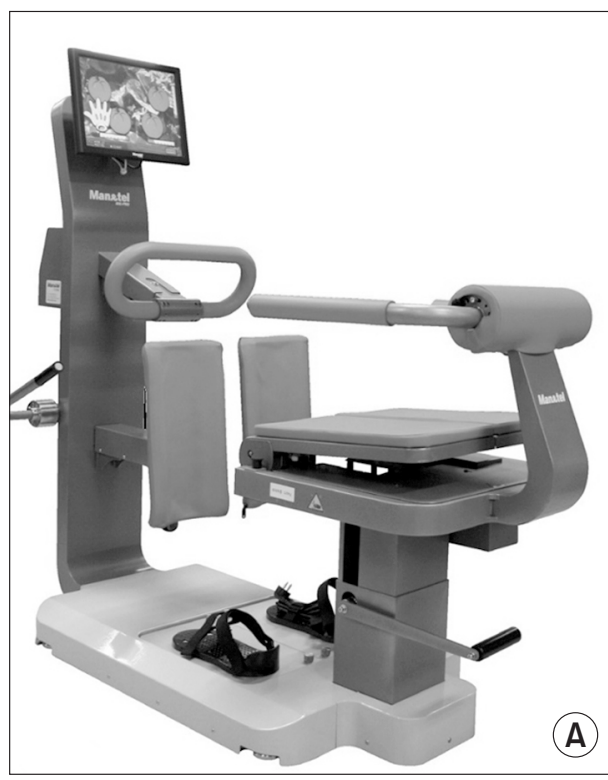

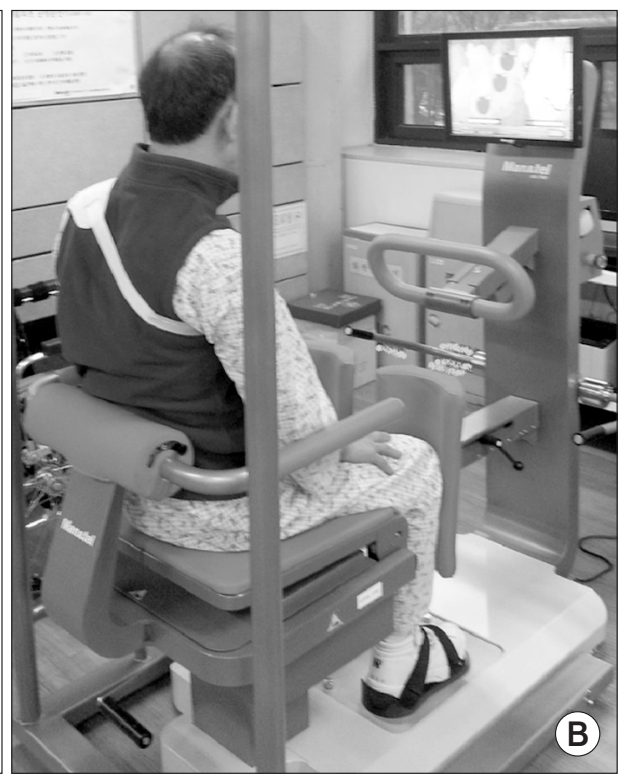

(B)
Fig. 1. (A, B) Trunk Stability Rehabilitation Robot Balance Trainer (Man\&tel, Gumi, Korea).
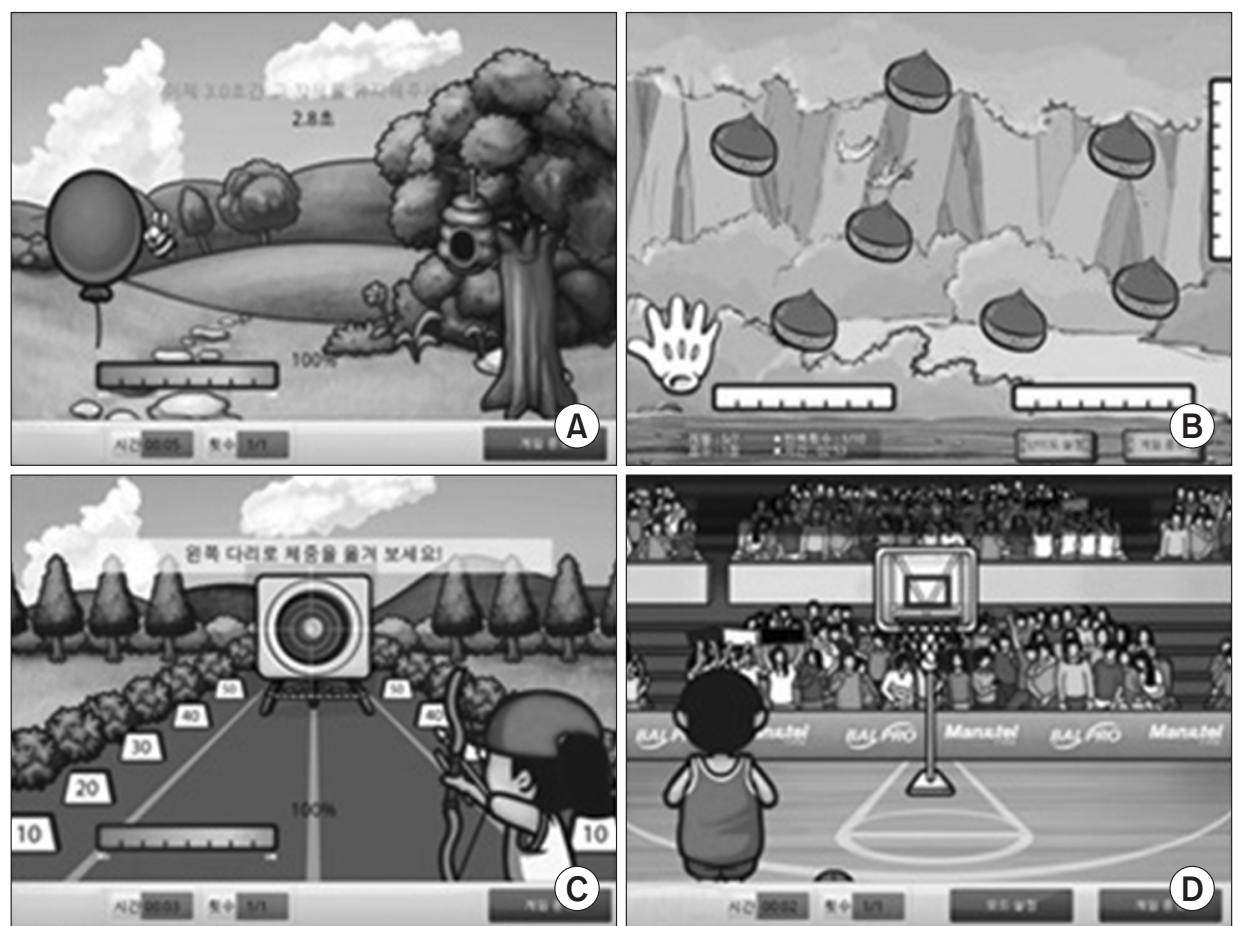

Fig. 2. (A) Balloon-popping game by moving the center of pressure (left/right). (B) Fruit-catching game by moving the hand icon according to the movement of the center of pressure (left/right and anterior/posterior). (C) Bull's eye matching game by moving the center of pressure, followed by extending the patient's arm beyond arm's length. (D) Basketball game by sit-to-stand movement assisted by chair section of the Trunk Stability Rehabilitation Robot Balance Trainer.

the patients proceeded with the TCT without the supervision of the therapist.

\section{Outcome measures}

The patients were evaluated before and after the 4-week intervention. We used the Trunk Impairment Scale (TIS) to assess trunk control function. The TIS consists of 3 subscales of static sitting balance (TIS-S, score ranges from 0 to 7), dynamic sitting balance (TIS-D, score ranges from 0 to 10), and trunk coordination (TIS-C, score ranges from 0 to 6 ). The total TIS (TIS-T) score ranges from 0 to 23 points; the higher the score, the better the balance of the trunk. The reliability, validity, measurement error, and internal consistency of the TIS for stroke patients have been reported in previous studies $[5,13,14]$. In addition to the TIS, the following assessment methods were 
also used in this study: the Berg Balance Scale (BBS), the Functional Ambulation Category (FAC), and the Korean version of the Modified Barthel Index (K-MBI) [4,15-17].

\section{Statistical analysis}

Data analysis was conducted with SPSS version 21.0 for Windows (IBM, Armonk, NY, USA). Descriptive statistics were performed for the collected variables of the participants. Chi-squared tests were used to compare the demographic data between groups. The Wilcoxon signed-rank test was used to compare baseline and post-intervention data within each group. Comparison of the change after intervention between the two groups was done with the Mann-Whitney U test. A p-value $<0.05$ was considered statistically significant.

\section{RESULTS}

\section{Characteristics of the subjects}

A total of 38 patients (the male to female ratio, 14:4 in LDT and 15:5 in HDT) were included in the analysis. The mean age of all participants was $55.8 \pm 18.6$ years, and the mean score on the Korean version of the MiniMental Status Examination (K-MMSE) was 20.3 \pm 8.1 . No statistical difference was observed in the demographic data between the LDT and HDT groups. The baseline TIS (LDT and HDT: $9.7 \pm 5.5$ and 9.3 \pm 5.5 , respectively), BBS (25.6 \pm 11.9 and $22.9 \pm 15.7$, respectively), FAC $(2.0 \pm 1.1$ and $1.9 \pm 1.0$, respectively), and K-MBI $(40.3 \pm 19.1$ and $44.3 \pm 11.9$, respectively) scores were not statistically different between the two groups (Table 1).

\section{Functional gain after intervention within each group}

Table 2 shows the functional gain after the 4 -week intervention. Both LDT and HDT groups showed significant improvements in their TIS ( $\mathrm{p}=0.001$ and $\mathrm{p}<0.001)$, TIS$\mathrm{S}(\mathrm{p}=0.018$ and $\mathrm{p}=0.001)$, TIS-D $(\mathrm{p}=0.016$ and $\mathrm{p}<0.001)$, TIS-C ( $\mathrm{p}=0.046$ and $\mathrm{p}<0.001)$, BBS $(\mathrm{p}=0.001$ and $\mathrm{p}=0.001)$, FAC $(p=0.008$ and $p=0.033)$, and K-MBI $(p=0.027$ and $\mathrm{p}=0.001)$ scores after the intervention.

\section{Comparisons between the LDT and HDT groups}

The HDT group showed greater statistical improvements than the LDT group in terms of their TIS ( $p=0.001)$, TIS-S ( $p=0.044)$, TIS- $\mathrm{D}(\mathrm{p}=0.033)$, and TIS- $\mathrm{C}(\mathrm{p}=0.012)$ scores. Differences between the LDT and HDT groups in
Table 1. Demographic data of subjects

\begin{tabular}{lcc}
\hline \multicolumn{1}{c}{ Variable } & $\begin{array}{c}\text { LDT group } \\
(\mathbf{n = 1 8})\end{array}$ & $\begin{array}{c}\text { HDT group } \\
(\mathbf{n = 2 0})\end{array}$ \\
\hline Sex & & \\
Male & 14 & 15 \\
\hline Female & $57.6 \pm 17.3$ & $54.2 \pm 20.0$ \\
\hline Age (yr) & & \\
Type of stroke & $12(10: 2)$ & $13(12: 1)$ \\
\hline Hemorrhagic (M:F) & $6(4: 2)$ & $7(3: 4)$ \\
\hline Ischemic (M:F) & & \\
Location of lesion & $16(12: 4)$ & $18(13: 5)$ \\
\hline Supratentorial (M:F) & $2(2: 0)$ & $2(2: 0)$ \\
\hline Infratentorial (M:F) & $8.3 \pm 9.6$ & $9.7 \pm 8.4$ \\
\hline Duration (mo) & $20.3 \pm 8.4$ & $20.3 \pm 8.2$ \\
\hline K-MMSE & $9.7 \pm 5.5$ & $9.3 \pm 5.5$ \\
\hline TIS-total & $4.8 \pm 1.9$ & $4.7 \pm 1.9$ \\
\hline TIS-static sitting balance & $2.7 \pm 3.1$ & $2.9 \pm 2.8$ \\
\hline TIS-dynamic sitting balance & $2.2 \pm 1.7$ & $1.7 \pm 1.7$ \\
\hline TIS-trunk coordination & $25.6 \pm 11.9$ & $22.9 \pm 15.7$ \\
\hline BBS & $2.0 \pm 1.1$ & $1.9 \pm 1.0$ \\
\hline FAC & $40.3 \pm 19.1$ & $44.3 \pm 11.9$ \\
\hline K-MBI & & \\
\hline Val & & \\
\hline
\end{tabular}

Values are presented as number or means \pm standard deviation.

M, male; F, female; K-MMSE, Korean version of the MiniMental Status Examination; TIS, Trunk Impairment Scale; BBS, Berg Balance Scale; FAC, Functional Ambulatory Classification; K-MBI, Korean version of Modified Barthel Index.

terms of their BBS, FAC, and K-MBI scores did not reach statistical significance (Table 2).

\section{DISCUSSION}

The results of this study indicated that TCT with TSRRBT could be an additional treatment to the conventional rehabilitation therapy for chronic stroke patients with poor sitting balance. Furthermore, trunk control ability was more improved in high-dose TCT than in low-dose TCT.

Symmetry and steadiness are important components of balance. Symmetry is used to describe equal weight distribution, and steadiness means the ability to maintain a specific posture [18]. Proper control of the center of gravity and movement is required to maintain balance 
Table 2. Comparison of therapeutic effects between LDT and HDT groups

\begin{tabular}{|c|c|c|c|c|c|c|c|}
\hline & \multicolumn{3}{|c|}{ LDT group } & \multicolumn{3}{|c|}{ HDT group } & \multirow{2}{*}{$\begin{array}{c}\text { p-value } \\
\text { (LDT vs. } \\
\text { HDT) }\end{array}$} \\
\hline & Pre-training & Post-training & p-value & Pre-training & Post-training & p-value & \\
\hline TIS (score) & $9.7 \pm 5.5$ & $12.5 \pm 5.3$ & $0.001^{*}$ & $9.3 \pm 5.5$ & $15.3 \pm 4.1$ & $0.000^{*}$ & $0.001^{* *}$ \\
\hline TIS-S & $4.8 \pm 1.9$ & $5.5 \pm 1.7$ & $0.018^{*}$ & $4.7 \pm 1.9$ & $6.5 \pm 0.9$ & $0.001^{*}$ & $0.044^{* *}$ \\
\hline TIS-D & $2.7 \pm 3.1$ & $4.2 \pm 3.3$ & $0.016^{*}$ & $2.9 \pm 2.8$ & $5.5 \pm 2.6$ & $0.000^{*}$ & $0.033^{* *}$ \\
\hline TIS-C & $2.2 \pm 1.7$ & $2.8 \pm 1.8$ & $0.046^{*}$ & $1.7 \pm 1.7$ & $3.5 \pm 1.4$ & $0.000^{*}$ & $0.012^{* *}$ \\
\hline BBS (score) & $25.6 \pm 11.9$ & $32.6 \pm 12.2$ & $0.001^{*}$ & $22.9 \pm 15.7$ & $28.1 \pm 16.7$ & $0.001^{*}$ & 0.251 \\
\hline FAC (category) & $2.0 \pm 1.1$ & $2.7 \pm 1.1$ & $0.008^{*}$ & $1.9 \pm 1.0$ & $2.4 \pm 0.9$ & $0.033^{*}$ & 0.393 \\
\hline K-MBI (score) & $40.3 \pm 19.1$ & $51.4 \pm 20.7$ & $0.027^{*}$ & $44.3 \pm 11.9$ & $57.0 \pm 14.5$ & $0.001^{*}$ & 0.654 \\
\hline
\end{tabular}

Values are presented as means \pm standard deviation.

LDT, low-dose training; HDT, high-dose training; TIS, Trunk Impairment Scale; TIS-S, static sitting balance subscale of TIS; TIS-D, dynamic sitting balance subscale of TIS; TIS-C, trunk coordination subscale of TIS; BBS, Berg Balance Scale; FAC, Functional Ambulatory Classification; K-MBI, Korean version of Modified Barthel Index.

${ }^{*} \mathrm{p}<0.05$, significant difference pre-training vs. post-training in a group.

${ }^{* *} \mathrm{p}<0.05$, significant difference between groups.

[19]. Lee et al. [20] suggested that weight-shift training in the sitting position with a visual biofeedback device encouraged patients' adoption of the correct posture by providing visual biofeedback, and led to low trunk sway velocity, and improved sitting balance.

Dynamic stability, the ability to move within a given posture, is also an important factor of balance [18]. The lower limbs play an active role in supporting the body mass during seated dynamic motion. In static sitting, over $80 \%$ of the body weight is supported through the seat and the remainder, through the feet. However, when people reach for an object over arm's length, about $50 \%$ of the body weight is supported through the feet [21]. Dean and Shepherd [22] reported that the practice of reaching in the sitting position beyond arm's length, which was accompanied by the appropriate loading of the affected leg, can improve the sitting balance in stroke patients. The ability to reach further and faster appears to be directly linked to the improved ability to use the affected leg for support and balance. Cabanas-Valdes et al. [23] reported that reaching training while sitting can improve trunk control.

The TCT with TSRRBT contains training protocols that can improve the crucial factors of sitting balance as mentioned above. As we expected, the TIS, BBS, FAC, and KMBI scores were significantly improved after the 4-week TCT with TSRRBT, suggesting an improvement in balance and functional outcome (Table 2).

The recovery plateau can be prolonged beyond six months after stroke, and an improvement in motor function can be achieved with repetitive and functional exercises with high intensity and duration [24]. The repetition of individual exercises is very important for recovery after stroke [25]. Thus, enhancing patients' motivation and compliance is also important for a successful outcome [26]. TSRRBT intervention is performed through a video game to attract patients' interest and provide a greater amount of repetitions in treatment. Therefore, we used TSRRBT for TCT in this study. In each training session, the patients performed a repeat weight-shift and weightbearing training exercises in the sitting position for a maximum of 280 times. On average for each session it was 150 times.

However, some additional factors should be considered. First, TSRRBT was unable to detect if the patient was performing the weight-shift training exercises appropriately or was just doing compensatory trunk movements. To address this problem, we provided the patients instructions on how to correctly perform the exercises before the treatment started. Further research may be needed to determine whether a therapist's supervision affected the outcome of the treatment. Second, some patients complained that the game was somewhat boring. Developing various types of games may be needed to maximize the patients' interest.

To investigate the effect of treatment dose on sitting balance improvement, we divided the patients into the following groups: LDT and HDT groups. After 4 weeks of 
intervention, the scores on the TIS and its subscales were significantly increased in the HDT group compared with the LDT group (Table 2). This finding suggests that TCT with TSRRBT may be dose dependent in improving patients' sitting balance. This result is consistent with previous studies [27,28], which reported that repeated training is important to improve the functioning of chronic stroke patients.

After 4 weeks of training, notable improvements in the BBS, FAC, and K-MBI scores for both the LDT and HDT groups were also reported. The differences between the LDT and HDT groups for these measured outcomes were not statistically significant, however. This result may be due to the fact that they did not reflect the change in sitting balance as well as TIS did. BBS, FAC, and K-MBI contain items that evaluate not only sitting balance, but also other components of general functioning, such as gait and sphincter control. For example, only 1 item out of the 16 evaluation items of BBS directly assesses balance function in the sitting position. The remaining items mainly measure any position change, standing balance, and gait function. These factors may have influenced the interpretation of the intervention outcomes.

The TIS was suggested as an assessment method to evaluate trunk performance. But trunk performance was poorly reflected by the other conventional assessment methods, such as BBS or FAC. Verheyden et al. [5] suggested that the total TIS score and each of the three subscale scores had sufficient reliability and validity in clinical assessment. Our study was targeted to patients with poor sitting balance (baseline TIS score of all patients, 9.5 \pm 5.4 ), so TIS could be used as an appropriate tool to measure sitting balance improvement. Therefore, we were able to confirm the dose dependency of TSRRBT intervention through the TIS scores.

During the 4-week intervention period, serious adverse events, such as falls and fractures, did not occur. However, two patients complained of temporary low back pain during a TSRRBT session. In these cases, training was continued only after correcting the training posture and reducing the pain.

This study also has limitations. First, it had no control group that did not receive TSRRBT training; thus, evaluating the effectiveness of conventional physical therapy was difficult. Nevertheless, TCT with TSRRBT may be presumed to have a positive effect on improving trunk con- trol ability on the basis of the high scores obtained by the HDT group compared with the LDT group. Second, the natural recovery process for a stroke might have affected the outcome. To minimize such effects in this study, we only included chronic stroke patients. Third, the sample size was small, and the study population was limited to stroke patients from a single center. Thus, generalizing the results of this study is difficult. Future research should conduct studies with a larger number of patients, including acute, subacute, and chronic stroke patients. In addition to these limitations others included: (1) the lack of a long-term follow-up of the treatment effect; (2) the assessors who were not blinded; and (3) the lack of a measure of the outcomes' effect size.

In conclusion, TCT using TSRRBT could be an additional treatment for the conventional rehabilitation therapy of chronic stroke patients with poor sitting balance. HDT may provide more beneficial effects on improving patients' sitting balance than LDT did.

\section{CONFLICT OF INTEREST}

No potential conflict of interest relevant to this article was reported.

\section{REFERENCES}

1. Langhorne P, Bernhardt J, Kwakkel G. Stroke rehabilitation. Lancet 2011;377:1693-702.

2. Karatas M, Cetin N, Bayramoglu M, Dilek A. Trunk muscle strength in relation to balance and functional disability in unihemispheric stroke patients. Am J Phys Med Rehabil 2004;83:81-7.

3. Bohannon RW, Cassidy D, Walsh S. Trunk muscle strength is impaired multidirectionally after stroke. Clin Rehabil 1995;9:47-51.

4. Jung HY, Park BK, Shin HS, Kang YK, Pyun SB, Paik NJ, et al. Development of the Korean Version of Modified Barthel Index (K-MBI): multi-center study for subjects with stroke. J Korean Acad Rehabil Med 2007;31:28397.

5. Verheyden G, Nieuwboer A, Mertin J, Preger R, Kiekens C, De Weerdt W. The Trunk Impairment Scale: a new tool to measure motor impairment of the trunk after stroke. Clin Rehabil 2004;18:326-34.

6. Chang WH, Kim YH. Robot-assisted therapy in stroke 
rehabilitation. J Stroke 2013;15:174-81.

7. Kwakkel G. Impact of intensity of practice after stroke: issues for consideration. Disabil Rehabil 2006;28:82330.

8. Walker C, Brouwer BJ, Culham EG. Use of visual feedback in retraining balance following acute stroke. Phys Ther 2000;80:886-95.

9. Stoller O, Waser M, Stammler L, Schuster C. Evaluation of robot-assisted gait training using integrated biofeedback in neurologic disorders. Gait Posture 2012;35:595-600.

10. Jung KH, Ha HG, Shin HJ, Ohn SH, Sung DH, Lee PK, et al. Effects of robot-assisted gait therapy on locomotor recovery in stroke patients. J Korean Acad Rehabil Med 2008;32:258-66.

11. Huh JS, Lee YS, Kim CH, Min YS, Kang MG, Jung TD. Effects of balance control training on functional outcomes in subacute hemiparetic stroke patients. Ann Rehabil Med 2015;39:995-1001.

12. Hung JW, Yu MY, Chang KC, Lee HC, Hsieh YW, Chen PC. Feasibility of using tetrax biofeedback video games for balance training in patients with chronic hemiplegic stroke. PM R 2016;8:962-70.

13. Verheyden G, Vereeck L, Truijen S, Troch M, Herregodts I, Lafosse $\mathrm{C}$, et al. Trunk performance after stroke and the relationship with balance, gait and functional ability. Clin Rehabil 2006;20:451-8.

14. Verheyden G, Nieuwboer A, De Wit L, Feys H, Schuback B, Baert I, et al. Trunk performance after stroke: an eye catching predictor of functional outcome. J Neurol Neurosurg Psychiatry 2007;78:694-8.

15. Blum L, Korner-Bitensky N. Usefulness of the Berg Balance Scale in stroke rehabilitation: a systematic review. Phys Ther 2008;88:559-66.

16. Collen FM, Wade DT, Bradshaw CM. Mobility after stroke: reliability of measures of impairment and disability. Int Disabil Stud 1990;12:6-9.

17. Mehrholz J, Wagner K, Rutte K, Meissner D, Pohl M. Predictive validity and responsiveness of the functional ambulation category in hemiparetic patients after stroke. Arch Phys Med Rehabil 2007;88:1314-9.
18. Goldie PA, Bach TM, Evans OM. Force platform measures for evaluating postural control: reliability and validity. Arch Phys Med Rehabil 1989;70:510-7.

19. Huxham FE, Goldie PA, Patla AE. Theoretical considerations in balance assessment. Aust J Physiother 2001;47:89-100.

20. Lee SW, Shin DC, Song CH. The effects of visual feedback training on sitting balance ability and visual perception of patients with chronic stroke. J Phys Ther Sci 2013;25:635-9.

21. Dean C, Shepherd R, Adams R. Sitting balance I: trunk-arm coordination and the contribution of the lower limbs during self-paced reaching in sitting. Gait Posture 1999;10:135-46.

22. Dean CM, Shepherd RB. Task-related training improves performance of seated reaching tasks after stroke: a randomized controlled trial. Stroke 1997;28:722-8.

23. Cabanas-Valdes R, Cuchi GU, Bagur-Calafat C. Trunk training exercises approaches for improving trunk performance and functional sitting balance in patients with stroke: a systematic review. NeuroRehabilitation 2013;33:575-92.

24. Staubli P, Nef T, Klamroth-Marganska V, Riener R. Effects of intensive arm training with the rehabilitation robot ARMin II in chronic stroke patients: four singlecases. J Neuroeng Rehabil 2009;6:46.

25. Teasell R, Bayona N, Salter K, Hellings C, Bitensky J. Progress in clinical neurosciences: stroke recovery and rehabilitation. Can J Neurol Sci 2006;33:357-64.

26. Maclean N, Pound P, Wolfe C, Rudd A. Qualitative analysis of stroke patients' motivation for rehabilitation. BMJ 2000;321:1051-4.

27. Lohse KR, Lang CE, Boyd LA. Is more better? Using metadata to explore dose-response relationships in stroke rehabilitation. Stroke 2014;45:2053-8.

28. Hsieh YW, Wu CY, Lin KC, Yao G, Wu KY, Chang YJ. Dose-response relationship of robot-assisted stroke motor rehabilitation: the impact of initial motor status. Stroke 2012;43:2729-34. 